\title{
An Integrated Optimal Design for Guaranteed Cost Control of Motor Driving System with Uncertainty
}

\author{
Tianyi Zeng, Xuemei Ren, Yao Zhang, Guang Li, Member, IEEE, and Jing Na, Member, IEEE
}

\begin{abstract}
A plant/controller integrated design strategy based on the nested optimization strategy and guaranteed cost control for a motor driving system is developed. Because of the coupling between the plant design and the controller design, the conventional sequential design methods cannot guarantee an overall optimality for the controlled motor driving system. The integrated design strategy proposed in this paper aims to tackle this problem by simultaneously optimizing the controller design and plant design. The integrated design objective is to drive the largest load and guarantee the satisfactory robust control performance for reference tracking subject to parametric uncertainties. This is enabled by integrating a guaranteed cost controller into the integrated design strategy and improves the reliability of the control scheme. A novel combined performance index including the plant design objective and the control performance is developed as the integrated design cost function for the motor driving system. A nested optimization strategy is employed so that the optimality of the system can be calculated in an efficient and reliable way. Experimental results demonstrate the effectiveness of the proposed integrated design method.
\end{abstract}

Index Terms-Integrated design of plant/controller, Guaranteed cost control, Motor driving system, Nested optimization method.

\section{INTRODUCTION}

A $\mathrm{S}$ a kind of mechatronic system, motor driving systems have drawn wide attention due to the broad application field. Many advance control methods have been employed to achieve a satisfactory control performance, such as adaptive control [1] and switching control [2]. The conventional design procedure for a control system, especially for a motor driving system, proceeds sequentially and separately, that is, the plant design is followed by the controller design [3]-[6]. However, this conventional design procedure simplifies the design process at the expense of performance degradation [7] because the coupling of the plant and controller designs is not considered and the optimality of the whole system including the physical plant and the controller cannot be guaranteed. The control performance of a motor driving system can be significantly influenced by the plant design because of the complex system structure. To enhance the control performance of a motor

This work was supported by National Natural Science Foundation of China under Grant 61433003, Grant 61973274 and Grant 61973036. (Corresponding authors: Yao Zhang and Guang Li).

$\mathrm{T}$. Zeng and X. Ren are with the College of Automation, Beijing Institute of Technology, Beijing, 100081 China. (e-mail: tyzeng0525@ outlook.com; xmren@bit.edu.cn).

Y. Zhang and Guang $\mathrm{Li}$ are with Queen Mary University of London, London, E1 4NS, UK. (e-mail: yao.zhang@qmul.ac.uk; g.li@qmul.ac.uk).

J. Na is with the Faculty of Mechanical and Electrical Engineering, Kunming University of Science and Technology, Kunming 650500, China. (e-mail: najing25@163.com). driving system, the plant/controller integrated design method can be employed by integrating the controller design into the plant design, so that an overall optimality for both plant and controller can be achieved simultaneously.

The integrated design, also known as co-design, has found its applications in many areas. The main benefit of the integrated design method is that the coupling existing between the plant design and the controller design can be explicitly considered to obtain the overall system optimality so that the system performance can be improved [8]. To enhance the performance of a micro-positioner, the parameters of both plant and controller are used as the optimization variables in one performance index [9]. Similar methods have been adopted for large space structures [10], [11], such as space antennas [12], and curved shell structures [13]. [14] addresses the integrated design of a wind turbine, which is a motordriving system. In [15], several optimization methods were developed to solve the integrated design problem. It is shown that more computational effort is required for the iterative strategy than the developed integrated design strategy especially when the model uncertainties exist. An integrated design optimization method aims to resolve an optimization problem with a combined plant performance index and a controller performance index by optimizing the plant and the controller parameters simultaneously and explicitly incorporating coupling constraints of the plant and the controller. The integrated design performance index combining the plant performance index and the controller performance index normally results in a non-convex optimization problem, even though each separate performance indexes of the plant design and the controller design is convex respectively [16]. The plant/controller coupling effect in the integrated design problem was investigated in [17] which reveals that the optimization problem can be decoupled if a necessary condition is satisfied. For this reason, the combined integrated design problem cannot be solved efficiently using conventional methods, such as the sequential optimization and the iterative optimization, which do not account for the coupling and thus cannot ensure the optimal solution to converge to the system-level optimality efficiently [18]. To derive a reliable and efficient optimal solution of the integrated design method and reduce the complexity of the integrated design optimization problem, the proposed integrated design adopts the nested optimization strategy, which can integrate a controller optimization problem into a plant optimization [19]. The nested strategy, as known as the bilevel optimization, is a special optimization method where one optimization problem is embedded into another. It has been widely used in estimating the demand for transportation 
[20], profitability management [21], engineering truss design problem [22]. The bi-level programming model is introduced in [23] to solve the collaborative logistics network resource matching problem. Using the nested optimization method, the integrated design problem can be simplified for each loop so that the system-level optimum can be achieved efficiently with low computational load. Compared with the integrated design methods based on intelligence optimization algorithms, the integrated design based on the nested-optimization has much lower computation burden as evidenced in [17].

An important issue we aim to address in this paper is the parametric modelling uncertainties, which are commonly encountered in integrated design applications. Due to the complicated structure of the motor driving system, the parametric uncertainties can be nontrivial in its modeling development. The control performance can be degraded if the parametric uncertainties are not fully considered in an integrated design [24]. To deal with the parametric uncertainties of a motor driving system including the load, a guaranteed cost controller is developed and adopted in the integrated design scheme in this paper. The controller is motivated by the concept of the guaranteed cost control proposed in [25], which can stabilize a control system subject to uncertainties with guaranteed performance. The guaranteed cost control has the advantage of providing an upper bound of a given performance index and thus the controlled motor driving system performance degradation incurred by the uncertainties is guaranteed to be less than the worst case [26], [27]. This advantage of using guaranteed cost control to cope with uncertainties is inherited by the proposed integrated design approach so that the guaranteed overall plant/controller performance optimality can be achieved for a system subject to parametric uncertainties, which guarantees the reliability of the control scheme. This newly added benefit does not increase extra complexity of the integrated design compared with other existing design methods, since the guaranteed cost control law can be written in a closed analytic form and then integrated into the efficient nested optimization scheme to achieve the overall optimality. The computational complexity is comparable to the existing LQR-based integrated design [17]. Thus, the integrated design approach proposed in this paper extends the LQR-based integrated design, which does not explicitly cope with parametric uncertainties.

The main contributions of this paper are summarized as follows:

1) An optimization index combining both the plant design and the controller design objectives is established to address the plant/controller integrated design. By choosing the inertia moment of the load as the plant optimization variable, the largest load with the satisfactory control performance can be achieved.

2) The unknown disturbances and parametric uncertainties in the plant are addressed by developing a guaranteed cost control in the integrated design scheme. The proposed method has improved robustness and reliability compared with the conventional LQR-based integrated design.

3) A nested optimization scheme is built to solve the integrated optimization problem, such that the integrated

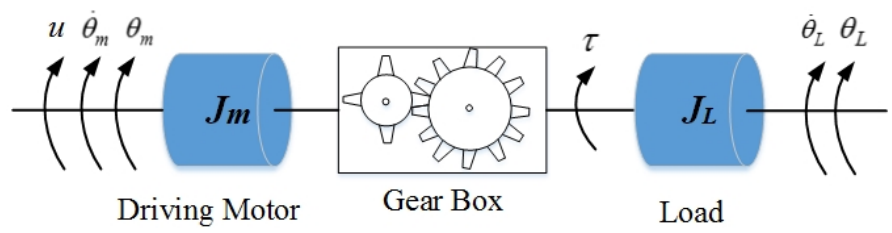

Fig. 1. Motor driving system

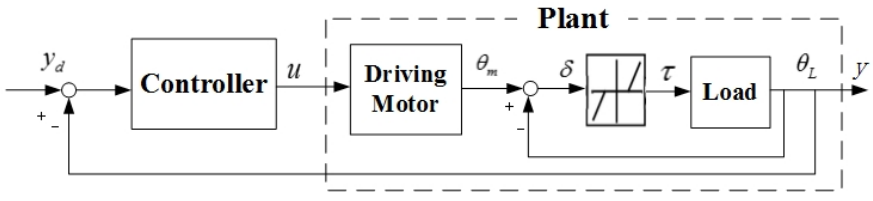

Fig. 2. Block diagram of a motor driving system

design problem can be simplified with economic computational efficiency.

The rest of this paper is organized as follows: In Section II, the model of a motor driving system is set up. A guaranteed cost robust controller is developed for the tracking control problem of the motor driving system in Section III. In Section IV, the optimization index combining the plant design and controller design is proposed for the integrated design problem of the motor driving system. A nested optimization strategy is employed to resolve the integrated design optimization problem reliably and efficiently. To illustrate the feasibility and effectiveness of the proposed strategy, experimental results of the motor driving system are presented in Section V. Finally, the paper is concluded in Section VI.

\section{Control-oriented Model Establishment}

In this section, control-oriented models for a motor driving system are presented. A motor driving system contains a driving motor and a load linked by a gear box as shown in Fig 1. The existence of the gear clearance is represented by a deadzone nonlinearity which is approximated by a combination of a linear term and a bounded term. The block diagram of the motor driving system is shown in Fig 2. The model can be described as:

$$
\left\{\begin{array}{l}
J_{m} \ddot{\theta}_{m}+b_{m} \dot{\theta}_{m}=u-\tau \\
J_{L} \ddot{\theta}_{L}+b_{L} \dot{\theta}_{L}=\tau
\end{array}\right.
$$

Here $\theta_{m}$ and $\theta_{L}$ are the angular positions of the driving motor and the load; $J_{m}$ and $J_{L}$ are moments of inertia of the driving motor and the load; $b_{m}$ and $b_{L}$ are the viscous friction coefficients of the driving motor and the load; $u$ is the control input; $\tau$ is the transmission torque of the gear box which can be expressed as

$$
\tau=k f(\delta)
$$

Here $k$ is the torsional coefficient; $\delta$ is the angle position error between the motor and the load, i.e. $\delta:=\theta_{m}-\theta_{L} ; f(\delta)$ is 
the deadzone nonlinearity expressed as

$$
f(\delta)= \begin{cases}\delta-\alpha, & \delta \geq \alpha \\ 0, & |\delta|<\alpha \\ \delta+\alpha, & \delta \leq-\alpha\end{cases}
$$

where $\alpha$ is the width of the gear clearance. To simplify the controller design, the deadzone nonlinearity is expressed as

$$
f(\delta)=\delta+d_{\alpha}(\delta)
$$

where

$$
d_{\alpha}(\delta)=\left\{\begin{array}{cc}
-\alpha, & \delta \geq \alpha \\
-\delta, & |\delta|<\alpha \\
\alpha, & \delta \leq-\alpha
\end{array}\right.
$$

Remark 1. From (4) and (5), the deadzone nonlinearity caused by the gear clearance is represented as a combination of a linear term and a saturated term satisfying $\left|d_{\alpha}(\delta)\right| \leq \alpha$. This can be treated as a parametric uncertainty later in the controller design and the integrated design procedure.

If system states are chosen as $x_{1}=\theta_{m}, x_{2}=\dot{\theta}_{m}, x_{3}=\theta_{L}$, $x_{4}=\dot{\theta}_{L}$, then the state-space model is

$$
\left\{\begin{array}{l}
\dot{x}_{1}=x_{2} \\
\dot{x}_{2}=-\frac{b_{m}}{J_{m}} x_{2}+\frac{1}{J_{m}} u-\frac{k}{J_{m}}\left(x_{1}-x_{3}\right)-\frac{k}{J_{m}} d_{\alpha}(\delta) \\
\dot{x}_{3}=x_{4} \\
\dot{x}_{4}=-\frac{b_{L}}{J_{L}} x_{4}+\frac{k}{J_{L}}\left(x_{1}-x_{3}\right)+\frac{k}{J_{L}} d_{\alpha}(\delta)
\end{array}\right.
$$

To incorporate the parametric uncertainties of the motor driving system, (6) can be modified as

$$
\left\{\begin{aligned}
\dot{x}_{1}= & x_{2} \\
\dot{x}_{2}= & -\left(a_{m 1}+\Delta a_{m 1}\right) x_{2}+\left(a_{u}+\Delta a_{u}\right) u \\
& \quad-\left(a_{m 2}+\Delta a_{m 2}\right)\left(x_{1}-x_{3}+d_{\alpha}(\delta)\right) \\
\dot{x}_{3}= & x_{4} \\
\dot{x}_{4}= & -\left(a_{L 1}+\Delta a_{L 1}\right) x_{4}+\left(a_{L 2}+\Delta a_{L 2}\right)\left(x_{1}-x_{3}+d_{\alpha}(\delta)\right)
\end{aligned}\right.
$$

Here, $a_{m 1}=\frac{b_{m}}{J_{m}}, a_{m 2}=\frac{k}{J_{m}}, a_{u}=\frac{1}{J_{m}}, a_{L 1}=\frac{b_{L}}{J_{L}}$, $a_{L 2}=\frac{k}{J_{L}} ; \Delta a_{m 1}, \Delta a_{m 2}, \Delta a_{u}, \Delta a_{L 1}$ and $\Delta a_{L 2}$ are bounded parametric uncertainties. Specifically, $\Delta a_{m 1}, \Delta a_{m 2}$ and $\Delta a_{u}$ stand for bounded parametric uncertainties existing in the driving motor; $\Delta a_{L 1}$ and $\Delta a_{L 2}$ stand for bounded parametric uncertainties existing in the load. Hence, parametric uncertainties along with the plant including the driving motor and the load are both taken into consideration.

The paper aims to use the plant/controller integrated design method to develop a tracking controller which can drive the system output $y=\theta_{L}$ to track a reference output $y_{d}$, subject to bounded parametric uncertainties and a deadzone nonlinearity, while at the same time maximizing the load to be driven. Note that the plant consists of the motor driving system and the load. The load's moment of inertia is considered as a design parameter of the plant in the integrated design.

\section{Controller Design}

In this section, a guaranteed cost controller is developed to tackle the tracking problem of the motor driving system with parametric uncertainties. In addition, the developed controller will be embedded in the integrated design scheme in the next section to drive the largest load in order to achieve the system level optimum. According to (7), the system model with parametric uncertainties can be rewritten concisely as

$$
\dot{x}=A x+B u_{c}+\Delta f(x)
$$

Here $A=\left[\begin{array}{cccc}0 & 1 & 0 & 0 \\ -a_{m 2} & -a_{m 1} & a_{m 2} & 0 \\ 0 & 0 & 0 & 1 \\ a_{L 2} & 0 & -a_{L 2} & -a_{L 1}\end{array}\right], B=\left[\begin{array}{c}0 \\ a_{u} \\ 0 \\ 0\end{array}\right]$ and $\Delta f=\left[\begin{array}{llll}0 & \Delta f_{1} & 0 & \Delta f_{2}\end{array}\right]^{T}$ is the lumped parametric uncertainty with $\Delta f_{1}=\Delta a_{u} u_{c}-\Delta a_{m 1} x_{2}-\Delta a_{m 2}\left(x_{1}-x_{3}+d_{\alpha}(\delta)\right)$ and $\Delta f_{2}=\Delta a_{L 2}\left(x_{1}-x_{3}+d_{\alpha}(\delta)\right)-\Delta a_{L 1} x_{4} ; u_{c}$ is the guaranteed cost control law to be designed. Throughout this paper, we assign an explicit structure to parametric uncertainties with the following assumption:

Assumption 1. Assume that parametric uncertainties $\Delta f(x)$ can be described as follows:

$$
\Delta f(x)=G d(x)
$$

where

$$
d^{T}(x) d(x) \leq x^{T} \xi x
$$

Here, $G=\operatorname{diag}\{0,1,0,1\}$ and $x^{T} \xi x$ are determined by the structure of the lumped uncertainty. $d(\cdot)$ is a function satisfying $d(0)=0$ and $\xi$ is a positive definite matrix relevant to the bound of parametric uncertainties [27].

The tracking error of the motor driving system $e$ is defined as $e:=y_{d}-\theta_{L}$. Considering the following performance index

$$
J=\int_{t_{0}}^{t}\left(e^{T} Q e+u_{c}^{T} R u_{c}\right) d t
$$

Here $Q$ and $R$ are positive constants. The Hamiltonian function for (8) with the performance index (11) is

$$
H\left(x, u_{c}\right)=e^{T} Q e+u_{c}^{T} R u_{c}+\lambda^{T}\left(A x+B u_{c}+\Delta f(x)\right)
$$

Definition 1. If there exists a control input $u_{c}$ and a desirable cost $J^{*}$ such that

1) the system (8) is stable, and

2) the cost function value satisfies $J \leq J^{*}$,

then $J^{*}$ is called a guaranteed cost for system (8). Furthermore, $u_{c}$ is called the corresponding guaranteed cost control input [28].

To investigate the parametric uncertainties and develop the guaranteed cost control, we present the following lemma:

Lemma 1. For the system model with parametric uncertainties (7), and the guaranteed cost control input $u_{c}$, we have

$$
\lambda^{T} \Delta f(x) \leq \Xi(x)
$$

with

$$
\Xi(x)=x^{T} \xi x+\frac{1}{4} \lambda^{T} G G^{T} \lambda
$$

Here $\lambda$ is the vector of Lagrange multiplier. 
Proof.

$$
\begin{aligned}
& \Xi(x)-\lambda^{T} \Delta f(x) \\
& =x^{T} \xi x+\frac{1}{4} \lambda^{T} G G^{T} \lambda-\lambda^{T} \Delta f(x) \\
& \geq d^{T}(x) d(x)+\frac{1}{4} \lambda^{T} G G^{T} \lambda-\lambda^{T} \Delta f(x) \\
& =\left(d(x)-\frac{1}{2} G^{T} \lambda\right)^{T}\left(\left(d(x)-\frac{1}{2} G^{T} \lambda\right) \geq 0\right.
\end{aligned}
$$

Hence $\lambda^{T} \Delta f(x) \leq \Xi(x)$.

Remark 2. As shown in [27], Lemma 1 provides $\Xi(x)$ with a specific form (14) which satisfies the following

$$
e^{T} Q e+u_{c}^{T} R u_{c}+\lambda^{T}\left(A x+B u_{c}\right)+\Xi(x)=0
$$

where the Lagrange multiplier $\lambda$ as introduced in Lemma 1 stands for the partial derivative of the cost function $J^{*}$ with respect to $x$, i.e. $\lambda=\partial J^{*} / \partial x$.

Next, to establish the relationship between $J$ defined by (11) and the guaranteed cost $J^{*}$ defined in Definition 1 , we consider the nominal system (7) without parametric uncertainties

$$
\left\{\begin{array}{l}
\dot{x}=A x+B u_{c} \\
y=C x
\end{array}\right.
$$

with the following performance index

$$
J^{*}\left(x\left(t_{0}\right), u_{c}\right)=\int_{t_{0}}^{t}\left(e^{\mathrm{T}} Q e+u_{c}^{\mathrm{T}} R u_{c}+\Xi(x)\right) d \varsigma
$$

and its corresponding Hamiltonian function is

$$
H^{*}\left(x, u_{c}\right)=e^{T} Q e+u_{c}^{T} R u_{c}+\Xi(x)+\lambda^{T}\left(A x+B u_{c}\right)
$$

From the necessary optimality conditions

$$
\left\{\begin{array}{l}
\frac{\partial H^{*}}{\partial u_{c}}=0 \\
\dot{\lambda}=-\frac{\partial H^{*}}{\partial x}
\end{array}\right.
$$

we have the control law

$$
u_{c}=-\frac{1}{2} R^{-1} B^{T} \lambda
$$

and

$$
\left[\begin{array}{c}
\dot{x} \\
\dot{\lambda}
\end{array}\right]=\left[\begin{array}{cc}
A & -B R^{-1} B^{T} \\
-2 C^{T} Q C-2 \xi & -A^{T}
\end{array}\right]\left[\begin{array}{l}
x \\
\lambda
\end{array}\right]+\left[\begin{array}{c}
0 \\
2 C^{T} Q y_{d}
\end{array}\right]
$$

$\lambda$ can be computed by [29],

$$
\lambda=P x-P_{t}
$$

Here, $P$ and $P_{t}$ are positive definite matrices and can be resolved from the algebraic Riccati equation shown as follows:

$$
\begin{gathered}
A^{T} P+P A-P B R^{-1} B^{T} P+2 C^{T} Q C+2 \xi=0 \\
P_{t}=2\left(P B R^{-1} B^{T}-A^{T}\right)^{-1} C^{T} Q y_{d}
\end{gathered}
$$

The following theorem aims to prove that $J^{*}$ is a guaranteed cost for the system (8) satisfying Definition 1.

Theorem 1. $J^{*}$ defined by (18) is a guaranteed cost for the system (8) with respect to $J$ defined by (11).
Proof. A Lyapunov candidate is chosen as

$$
V=J^{*}\left(x\left(t_{0}\right), u_{c}\right)
$$

Then,

$$
\dot{V}=\frac{\partial V}{\partial x} \dot{x}=\lambda^{T} \dot{x}=\lambda^{T}\left(A x+B u_{c}+\Delta f(x)\right)
$$

According to Lemma 1,

$$
\dot{V} \leq \lambda^{T}\left(A x+B u_{c}\right)+\Xi(x)
$$

According to Remark 2, we have

$$
\dot{V} \leq-e^{T} Q e-u_{c}^{T} R u_{c}<0
$$

Hence, the system is asymptotically stable with the designed controller. Next, we show that $J^{*}$ is an upper bound of $J$ subject to the nominal system (17).

Considering $J^{*}$ in (18), we have

$$
\dot{J}^{*}=\frac{\partial J^{*}}{\partial x} \frac{\partial x}{\partial t}=\lambda^{T}\left(A x+B u_{c}+\Delta f(x)\right)
$$

Then

$$
\begin{aligned}
0= & -\dot{J}^{*}+\lambda^{T}\left(A x+B u_{c}+\Delta f(x)\right) \\
e^{T} Q e+u_{c}^{T} R u_{c}= & -\dot{J}^{*}+\lambda^{T}\left(A x+B u_{c}+\Delta f(x)\right) \\
& +e^{T} Q e+u_{c}^{T} R u_{c}
\end{aligned}
$$

From Lemma 1, we have $\lambda=\partial J^{*} / \partial x$. Then,

$$
\begin{aligned}
e^{T} Q e+u_{c}^{T} R u_{c} \leq & -\dot{J}^{*}+\lambda^{T}\left(A x+B u_{c}\right)+\Xi(x) \\
& +e^{T} Q e+u_{c}^{T} R u_{c}
\end{aligned}
$$

According to Remark 2, we have

$$
e^{T} Q e+u_{c}^{T} R u_{c} \leq-\dot{J}^{*}
$$

Integrating (32) from $t_{0}$ to $t$ yields

$$
\int_{t_{0}}^{t}\left(e^{\mathrm{T}} Q e+u_{c}^{\mathrm{T}} R u_{c}\right) d \varsigma \leq-J^{*}(x(t))+J^{*}\left(x\left(t_{0}\right)\right)
$$

Since the system is asymptotically stable, with (11), we have

$$
J\left(x\left(t_{0}\right), u_{c}\right) \leq J^{*}\left(x\left(t_{0}\right), u_{c}\right)
$$

When $\Delta f(x)=0$, we have $J\left(x\left(t_{0}\right), u_{c}\right)=J^{*}\left(x\left(t_{0}\right), u_{c}\right)$. Summing up the above, the cost index (11) satisfies the following inequality

$$
J\left(x\left(t_{0}\right), u_{c}\right) \leq J^{*}\left(x\left(t_{0}\right), u_{c}\right)
$$

Remark 3. According to Definition 1, $u_{c}$ designed for the nominal system (17) with the cost function (18) provides a guaranteed cost control for the system (8) with the cost function (11). Hence, the reliability design of the control system can be achieved by the proposed guaranteed cost control. 


\section{Plant/Controller Integrated Design Method}

In this section, a combined integrated design optimization index is established and the coupling between the plant and controller optimization problems is analyzed. Then, the guaranteed cost control is embedded into a nested optimization strategy to solve the integrated design problem so that the largest load which can be driven with the satisfactory control performance is achieved. Finally, it is proved that the overall system-level optimality can be guaranteed by the nested optimization strategy.

For the system (7), we choose the load's moment of inertia $J_{L}$ as a design parameter for plant design and (18) as the control optimization index. A combined controller/plant integrated design optimization index is [18]:

$$
\begin{aligned}
& \chi=\min _{J_{L}, P}\left\{w_{p} f_{p}+w_{c} f_{c}\right\} \\
& \text { s.t. } g\left(J_{L}\right) \leq 0, h\left(J_{L}\right)=0
\end{aligned}
$$

Here $w_{p}$ and $w_{c}$ are weights reflecting the trade-off between the emphasis on the plant design and the controller design; $h\left(J_{L}\right)=0$ is the equality constraint including system dynamics; $g\left(J_{L}\right)$ is the inequality constraint and for this case it can be expressed as $J_{\text {low }} \leq J_{L} \leq J_{\text {high }} ; f_{p}=\frac{1}{J_{L}}$ and $f_{c}=\int_{t_{0}}^{t}\left(e^{\mathrm{T}} Q e+u_{c}^{\mathrm{T}} R u_{c}+\Xi(x)\right) d \varsigma$ are the plant and control optimization objectives, respectively. The optimal solution of (37) is expressed as

$$
\Phi^{*}=\arg \min _{J_{L}, P} \chi
$$

Remark 4. The purpose of the integrated design optimization problem (37) is to seek the largest inertia moment of the load without degrading the control performance.

Remark 5. All the system states are assumed to be measurable; otherwise, a state observer needs to be designed. The weights $w_{p}$ and $w_{c}$ are determined by different integrated design scenarios. By tuning $w_{p}>w_{c}$, a larger load can be achieved along with the increase of the control input; On the contrary, if we selected $w_{p}<w_{c}$, a smaller load can be achieved with a smaller control cost, i.e. a smaller torque required from the driving motor. Once they are determined, the integrated design approach aims to find the optimal solution for both of the plant design and the controller design. The weights for the integrated design of a motor driving system can be determined by the requirement of reference tracking performance, the maximum load and other hardware specifications such as the power and cost of motors. A sample motor selection for different scenario can be referred by the integrated design results which indicate the feasibility of the developed mechatronic system. Relevant results are illustrated in Section V.

Note that a sequential design strategy cannot guarantee the system-level optimality because the coupling of the plant design and controller design cannot be taken into account [17]. A necessary condition for decoupling these two designs is developed in [17]. It shows that for the two designs in a sequential approach to be decoupled, the weights must satisfy $\frac{w_{c}}{w_{p}} \rightarrow 0$, which means that the plant objective is infinitely more important than the controller objective. To overcome this issue, we develop an integrated design method where the weight parameters can be chosen based on practical scenarios and a guaranteed cost can be achieved for a system subject to bounded parametric uncertainties. Specifically, an integrated design method based on the nested optimization and the guaranteed cost control to solve the problem of (37) is proposed as follow:

Outer Loop:

$$
\begin{aligned}
& J_{L}^{*}=\arg \min \left\{w_{p} \frac{1}{J_{L}}+w_{c} f_{c}^{*}\left(J_{L}\right)\right\} \\
& \text { s.t. } J_{\text {low }} \leq J_{L} \leq J_{\text {high }}, \\
& \quad f_{c}^{*}\left(J_{L}\right)=\text { results of the inner loop. }
\end{aligned}
$$

Inner Loop:

$$
\begin{aligned}
& f_{c}^{*}\left(J_{L}\right)=\min \int_{t_{0}}^{t}\left(e^{\mathrm{T}} Q e+u_{c}^{\mathrm{T}} R u_{c}+\Xi(x)\right) d \varsigma \\
& \text { s.t. } \dot{x}=A x+B u_{c}, \quad x\left(t_{0}\right)=x_{0} .
\end{aligned}
$$

Here, $u_{c}$ is the guaranteed cost control designed in Section III:

$$
\begin{aligned}
u_{c}^{*} & =-\frac{1}{2} R^{-1} B^{T} \lambda, \\
\lambda & =P x-P_{t} \\
0 & =A^{T} P+P A-P B R^{-1} B^{T} P+2 C^{T} Q C+2 \xi \\
P_{t} & =2\left(P B R^{-1} B^{T}-A^{T}\right)^{-1} C^{T} Q y_{d}
\end{aligned}
$$

Remark 6. The outer loop is for the plant optimization; the inner loop is for the controller optimization with feasible plant design chosen by the outer loop. The proposed integrated design scheme ensures the overall system-level optimality can be achieved. Note that the plant optimization is resolved in the outer loop; otherwise, its solution is always the largest value of $J_{L}$ within the constraints, which is not desirable.

To illustrate the implementation of the proposed integrated design method, a flow chart is presented in Fig. 3(a). For comparison, a schematic diagram of the iterative design method is shown in Fig. 3(b). 


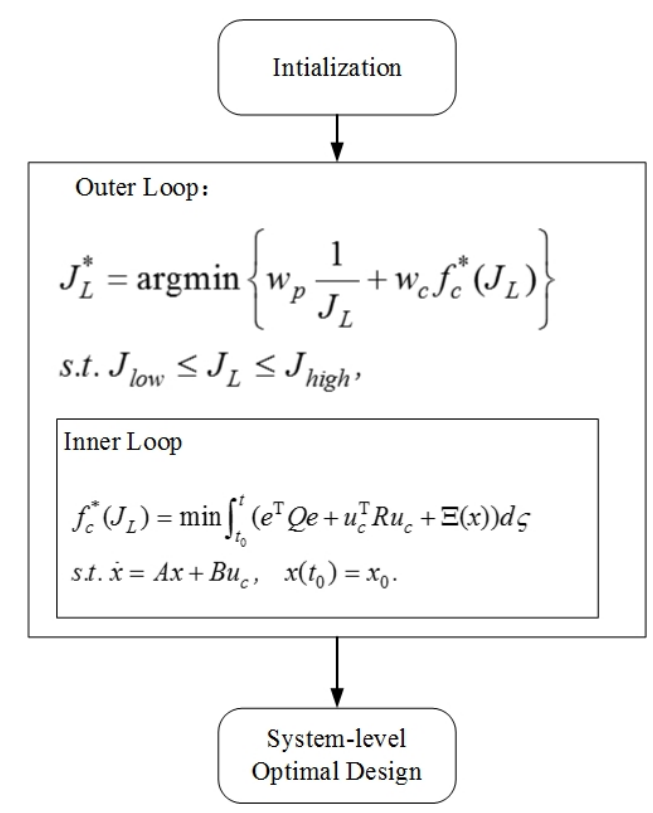

(a) Integrated design with nested optimization strategy

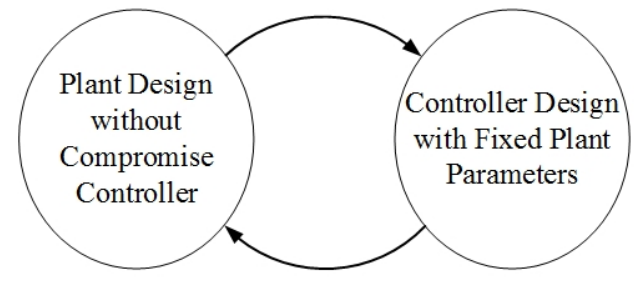

(b) Iterative design procedure

Fig. 3. Flowcharts of nested integrated design and iterative design

As shown in Fig 3, reiteration the controller design and the plant design with a convergence index, the iterative design can only guarantee the optimality of subsystems. By introducing the proposed method, the dynamic optimization (inner loop) is embedded into the static optimization (outer loop), which obtains the system-level optimality because both the plant design and the controller design are considered simultaneously. In addition, the separation achieved by the two loops makes each of them can be solved using accordingly befitting methods [30].

The following definitions are to be used later in Theorem 2 and its proof.

Definition 2. $\Psi$ is a set of feasible solutions of the nested optimization method.

Definition 3. For a given controller, there is a set of feasible solutions for the plant $\Psi_{p}(P)=\left\{J_{L}:\left(J_{L}, P\right) \in \Psi\right\}$.

Definition 4. For a given plant, there is a set of feasible solutions for the controller $\Psi_{c}\left(J_{L}\right)=\left\{P:\left(J_{L}, P\right) \in \Psi\right\}$.

The following theorem guarantees that the overall optimality for the controlled motor driving system can be achieved by the integrated design proposed in this paper, i.e. (39) and (40).

Theorem 2. Let $\Psi^{*}$ be the solution of the nested optimization method proposed in (39) and (40). Then $\Psi^{*}=\Phi^{*}$.
Proof. $\forall\left(J_{L}, P\right), J_{L} \in \Psi_{p}$ and $P \in \Psi_{c}\left(J_{L}\right)$, with the Definitions 3 and $4, J_{L} \in \Psi_{p}(P)$. Thus, $\Psi_{p}=\Psi\left(P^{*}\right)$ and (39) can be modified as

$$
f_{p}^{*}=\min _{J_{L} \in \Psi_{p}\left(f_{c}^{*}\right)}\left\{w_{p} \frac{1}{J_{L}}+w_{c} f_{c}^{*}\left(J_{L}\right)\right\}
$$

Define the optimal plant/controller solutions of (37) respectively as

$$
\begin{gathered}
J_{L}^{*}=\arg \min _{J_{L} \in \Psi_{p}\left(P^{*}\right)} \chi\left(J_{L}, P^{*}\right) \\
P^{*}=\arg \min _{P \in \Psi_{c}\left(J_{L}^{*}\right)} \chi\left(J_{L}^{*}, P\right)
\end{gathered}
$$

Then we have $\left(J_{L}^{*}, P^{*}\right) \in \Psi^{*}$. According to (40), for the optimality of the plant $J_{L}^{*}$, we have $P^{*}=f_{c}^{*}\left(J_{L}^{*}\right)$ in (43) and (44). Then

$$
J_{L}^{*}=\arg \min _{J_{L} \in \Psi_{p}\left(P^{*}\right)} \chi\left(J_{L}, f_{c}^{*}\left(J_{L}^{*}\right)\right),
$$

which is (39). Thus,

$$
\left(J_{L}^{*}, P^{*}\right) \in \Phi^{*} \forall\left(J_{L}^{*}, P^{*}\right) \in \Psi^{*}
$$

which means $\Psi^{*} \subseteq \Phi^{*}$.

Suppose that $\left(J_{L}^{*}, P^{*}\right) \in \Phi^{*}$, then $\left(J_{L}^{*}, P^{*}\right)$ satisfies (43) and (44). Since $f_{c}^{*}\left(J_{L}^{*}\right)=P^{*}$, (43) can be modified as

$$
\min _{J_{L}\left(P^{*}\right)}\left\{w_{p} \frac{1}{J_{L}}+w_{c} f_{c}^{*}\right\}
$$

which is (43). Then,

$$
\left(J_{L}^{*}, P^{*}\right) \in \Psi^{*} \forall\left(J_{L}^{*}, P^{*}\right) \in \Phi^{*}
$$

Thus we have $\Phi^{*} \subseteq \Psi^{*}$.

Therefore, we have $\Phi^{*}=\Psi^{*}$, which completes the proof.

Remark 7. With the integrated design method based on the nested optimization method, the plant/controller integrated design problem can be divided into a constrained nonlinear programming (NLP) problem for the outer loop and a guaranteed cost control in the inner loop. A constrained NLP problem can be solved by many existing software packages. In this paper, we choose 'fmincon' in MATLAB.

Remark 8. With the integrated design scheme proposed above, the complexity of combined plant/controller optimization problems can be reduced without compromising the overall system optimality [17]. The integrated design problem is simplified for each loop so that the system-level optimality can be obtained which makes the feasibility of the control system can be further guaranteed.

\section{EXPERIMENTAL VERIFICATION}

To experimentally validate the effectiveness of the proposed integrated design method, a motor driving system test rig is established as shown in Fig. 4. 
TABLE I

SySTEM PARAMETERS

\begin{tabular}{ccc} 
Parameter & Value & Unit \\
\hline$J_{m}$ & 0.026 & $\mathrm{~kg} \cdot \mathrm{m}^{2}$ \\
$b_{m}$ & 1.3 & $\mathrm{Nm} \cdot \mathrm{s} / \mathrm{rad}$ \\
$b_{l}$ & 1.3 & $\mathrm{Nm} \cdot \mathrm{s} / \mathrm{rad}$ \\
$\alpha$ & 0.2 & $\mathrm{rad}$ \\
$k$ & 560 & $\mathrm{Nm} / \mathrm{rad}$ \\
\hline
\end{tabular}

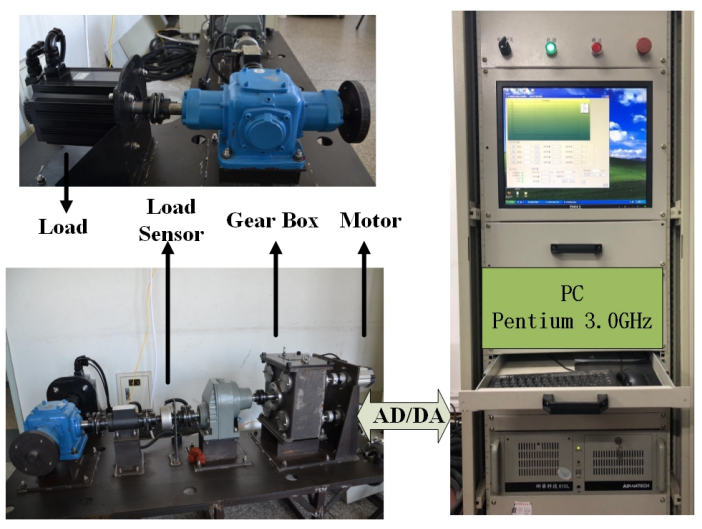

Fig. 4. Motor driving system setup

The system comprises a driving motor with the pulse width modulation (PWM) amplifier located in the driving card (Panasonic MCDDT3520), an electromotor (180ST-M 35105) with 64000 counts per rotation resolution encoder as the load, a digital signal processing (TMS320 2812) unit performing for communication, and a Pentium $3.0 \mathrm{GHz}$ industrial control computer by running $\mathrm{C}++$ program in CCS 5.0 developing environment. The load is driven by the driving motor through the transmission gear. The industrial computer receives the signals of the driving motor and load from the encoder, and provides the corresponding control action for real-time control. The sampling time is $t_{s}=0.001 \mathrm{~s}$. A sinusoidal signal $y_{d}=10 \sin \left(\frac{2}{5} \pi t\right) \mathrm{rad}$ is chosen as a reference signal. The system parameters are shown in the Table I. Because system parameters of the test rig are not exactly known, values in Table I are given by estimations based on our experiences, which inevitably introduces parametric uncertainties. According to the estimations, parametric uncertainties vary within $5 \%$ of the nominal values of the parameters shown in Table I. The load's moment of inertia can be adjusted by adding additional load components. The detailed parameter tunings are as follows: Select the weight parameters as $w_{p}=w_{c}=1$, plant constraints as $J_{\text {low }}=0.009 \mathrm{~kg} \cdot \mathrm{m}^{2}$ and $J_{\text {high }}=0.556 \mathrm{~kg} \cdot \mathrm{m}^{2}$, and the guaranteed cost controller parameters $Q=30, R=1$ and $\xi=\operatorname{diag}\{0.05,0.5,0.05,0.5\}$. Note that the values of $w_{p}$ and $w_{c}$ chosen here are merely for demonstration purpose, and experimental validation can be done with different values. In the following, an existing integrated design approach [17] based on the LQR control is tested for comparison with the proposed integrated design with the guaranteed cost control. A Monte Carlo analysis is also presented to show the effectiveness of the proposed control law for different initial states. Comparative results are given to

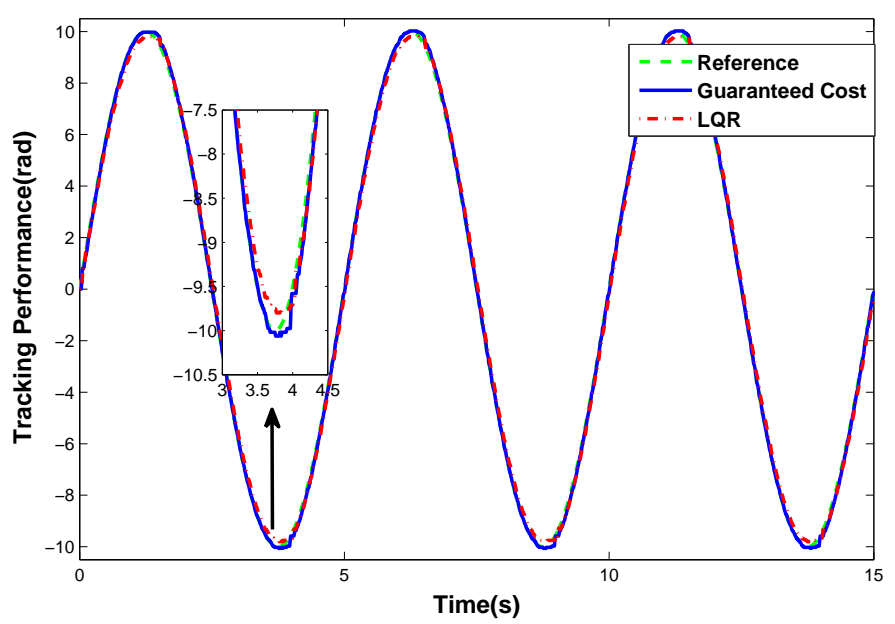

Fig. 5. Tracking performance with guaranteed cost control and LQR
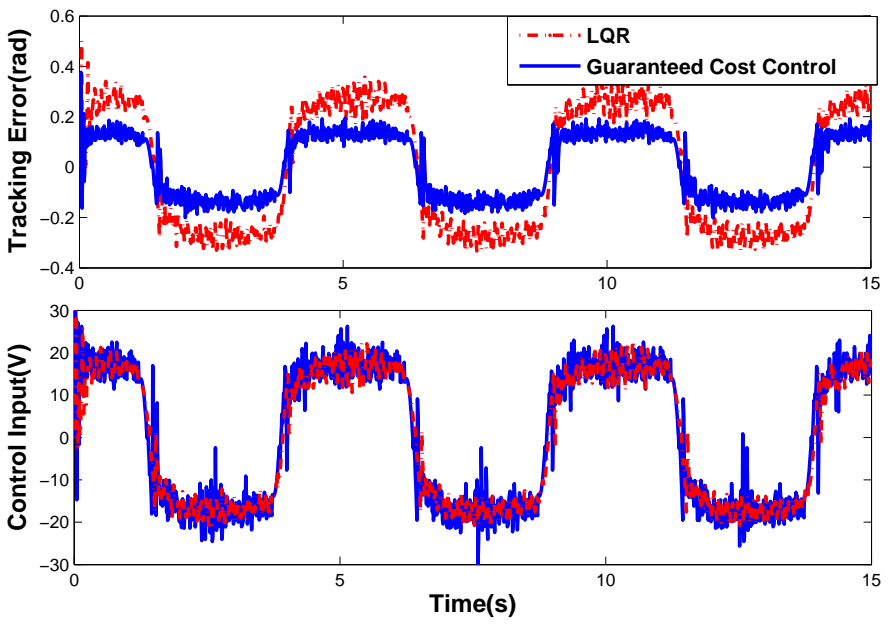

Fig. 6. Tracking error and control input with guaranteed cost control and LQR

demonstrate the effectiveness of the integrated design scheme.

\section{A. Controller Verification}

Using the integrated design method raised in this paper, we can derive $J_{L}^{*}=0.3233 \mathrm{~kg} \cdot \mathrm{m}^{2}$ and $u_{c}=-k_{c} x+1.2 y_{d}$ where $k_{c}=\left[\begin{array}{llll}0.47 & 0.19 & 0.54 & 0.05\end{array}\right]$. For comparison purpose, we also test an existing integrated design approach based on the LQR control for this motor driving test rig [17]. The cost function in the inner loop is defined as (11). $J_{L}^{*}=0.3250 \mathrm{~kg} \cdot \mathrm{m}^{2}$ is achieved when using the LQR-based integrated design method with the same choice of weight parameters and constraints. Note that although a tiny larger load can be achieved by the LQR-based integrated design, the parametric uncertainty is not taken into consideration which degrades the control performance as shown in the following results. A comparison of tracking performance of the two integrated designs respectively based on the LQR controller and the guaranteed cost controller is shown in Fig. 5 and Fig. 6.

Fig. 5 and Fig. 6 show that when using the integrated design based on the guaranteed cost control, the motor driving system 


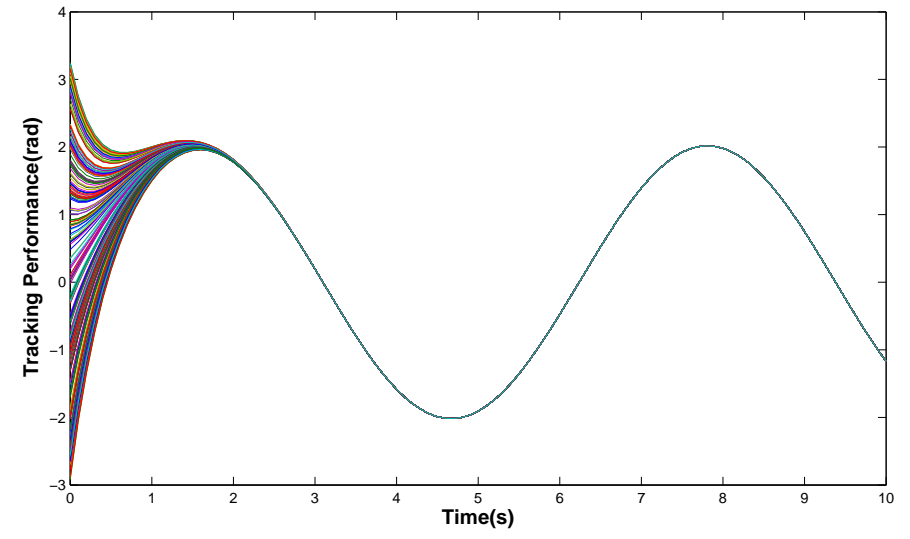

Fig. 7. Monte Carlo simulation with guaranteed cost control

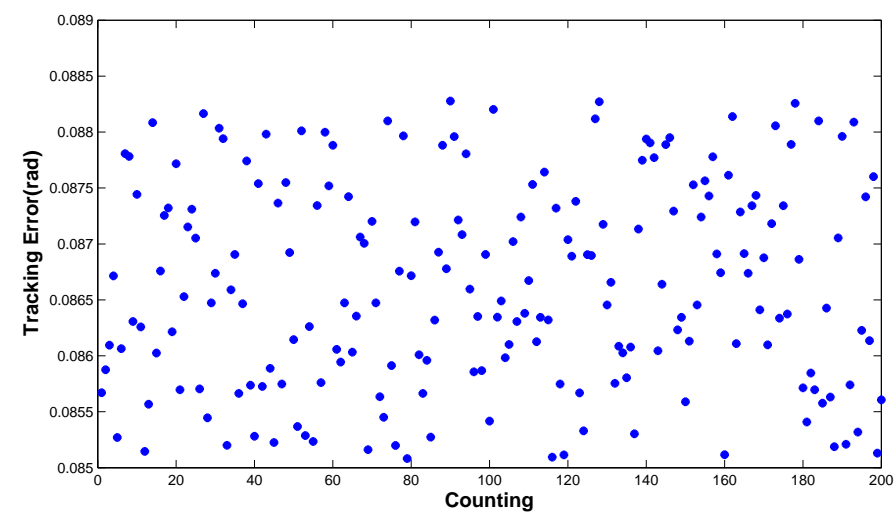

Fig. 8. Tracking performance at $t=3(s)$

can track the reference signal with satisfactory performance. Fig. 6 shows a larger tracking error when the LQR controller is employed designed in the integrated design method. The tracking performance of the LQR controller is more easily influenced by parametric uncertainties, whilst a better control performance can be obtained with the proposed integrated design based on the guaranteed cost controller.

To further show the robustness of the presented method, a numerical Monte Carlo analysis of 200 cases with different initial states based on the proposed integrated design approach is presented in Fig. 7 and Fig. 8. To verify the robustness of the proposed guaranteed cost control, a large parametric uncertainty is taken into consideration in the Monte Carlo analysis. The mean value of initial position is $0 \mathrm{rad}$, and the standard deviation of initial position is $3 \mathrm{rad}$; the mean value of initial velocity is $0 \mathrm{rad}$, and the standard deviation of initial velocity is $0.5 \mathrm{rad} / \mathrm{s}$. An time varying uncertainty $d(x)=0.2 \sin (t)$ is introduced to further testify the efficacy of the proposed controller.

Fig. 7 and Fig. 8 show that the largest tracking error is $0.0885 \mathrm{rad}$ and $90 \%$ tracking errors locate in the range of $[0.085,0.088] \mathrm{rad}$. Hence, the satisfactory control performance can be achieved with the initial perturbation and the timevarying parametric uncertainty which guarantees the reliability of proposed method.

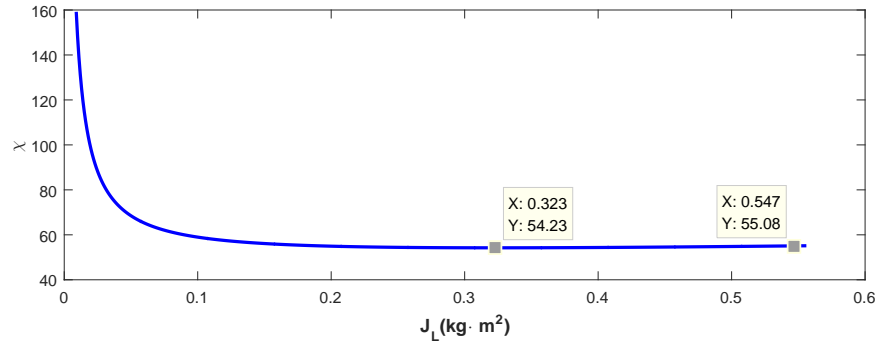

Fig. 9. Integrated design performance index
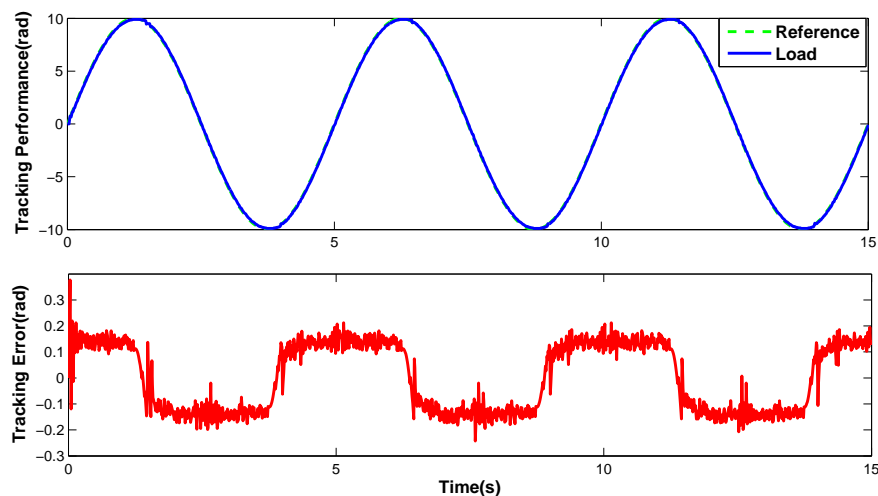

Fig. 10. Tracking performance with guaranteed cost controller when the load is changed

\section{B. Integrated Design Scheme Verification}

To verify the optimality of the plant/controller integrated design problem achieved using the proposed integrated design scheme in this paper, the optimal values of the combined cost function of (37) are shown in Fig. 9, from which we can see that $\chi$ is convex and the optimal solution is $J_{L}^{*}=0.3233 \mathrm{~kg} \cdot \mathrm{m}^{2}$. Theoretically, the tracking performance is influenced by the inertia moment of the load significantly. The tracking performance for the inertia moment of the load decreases from $J_{L}^{*}=0.3233 \mathrm{~kg} \cdot \mathrm{m}^{2}$ to $J_{L}=0.1130 \mathrm{~kg} \cdot \mathrm{m}^{2}$, as shown in Fig. 10. It can be also seen from Fig. 10 that with different moments of inertia of the load, the same tracking performance can be achieved. Note that $J_{L}=0.1130 \mathrm{~kg} \cdot \mathrm{m}^{2}$ is a frequently-used plant parameter. With the proposed integrated design method, the additional moment of inertia of the load can be driven without degrading tracking performance. As another advantage of the integrated design method developed in this paper, the weight parameters $w_{p}$ and $w_{c}$ can be tuned for different scenarios. The Pareto surface is shown in Fig. 11 for $w_{c}$ ranging from 1 to 100 and for $w_{p}$ from 1 to 10 . We can see that by tuning weight parameters, different optimal solutions of the integrated design can be achieved. To further investigate the influence of weight parameters, we choose $w_{c}=20$ and let $w_{p}$ change within the range of $[2,10]$. Control inputs with different weight parameters are shown in Fig 12.

Combining Fig 11 and Fig 12, we can see that by tuning $w_{p}>w_{c}$, a larger load can be achieved; however, a larger control input is needed. Specifically, in Fig 12, by choosing $w_{p}=2$ and $w_{p}=10$, the corresponding amplitude of the control input is $12 \mathrm{~V}$ and $21 \mathrm{~V}$, respectively, which leads a 


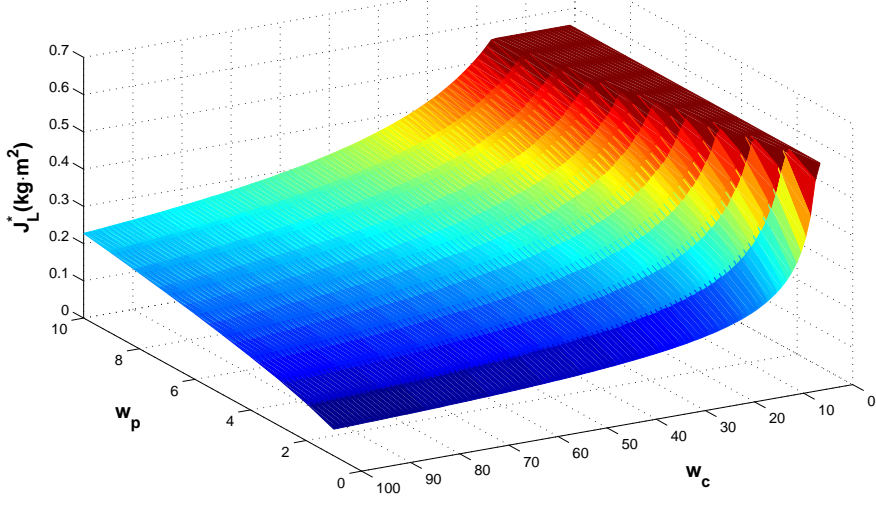

Fig. 11. Weight parameters Pareto surface

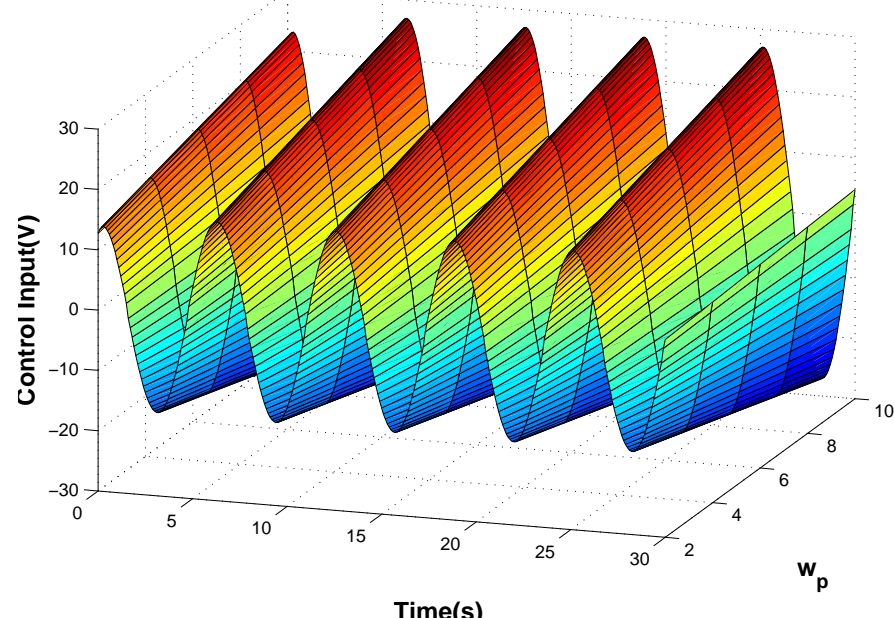

Fig. 12. Control inputs with different weight parameters

different torque requirement providing by the driving motor. A sample motor selection for different scenario can be referred by the integrated design results which indicate the torque requirement rating of the driving motor. This means that the initial system design, which is a control-optimal design, requires the use of a motor capable of providing such torque. In practical system setup, driving motors can be selected based on the co-design result which makes the reliability design can be further guaranteed for the mechatronic system. Hence, weight parameters can be tuned based on different practical requirements as stated in Remark 5.

\section{CONCLUSION}

A novel plant/controller integrated design method is developed for a motor driving system in this paper. A guaranteed cost tracking controller is embedded into the integrated design to deal with the parametric uncertainty of the motor driving system. To improve the tracking performance of the system, an optimization index combining both the plant and the controller is built and solved using the nested optimization strategy. Using the proposed optimization strategy, the optimality of the integrated design method can be guaranteed by reasonably accounting for the relative importance between the plant and the controller. The efficacy of the proposed integrated design method is experimentally validated.

\section{REFERENCES}

[1] S. Wang, H. Yu, J. Yu, J. Na, and X. Ren, "Neural-network-based adaptive funnel control for servo mechanisms with unknown dead-zone," IEEE Transactions on Cybernetics, vol. PP, no. 99, pp. 1-12.

[2] Y. Zhu, Z. Zhong, M. V. Basin, and D. Zhou, "A descriptor system approach to stability and stabilization of discrete-time switched pwa systems," IEEE Transactions on Automatic Control, vol. 63, no. 10, pp. 3456-3463, 2018.

[3] R. Wang, H. Jing, F. Yan, H. R. Karimi, and N. Chen, "Optimization and finite-frequency $h_{\infty}$ control of active suspensions in in-wheel motor driven electric ground vehicles," Journal of the Franklin Institute, vol. 352, no. 2, pp. 468-484, 2015.

[4] R. J. Wai, "Total sliding-mode controller for pm synchronous servo motor drive using recurrent fuzzy neural network," IEEE Transactions on Industrial Electronics, vol. 48, no. 5, pp. 926-944, 2001.

[5] Y. Zhang, R. Vepa, G. Li, and T. Zeng, "Mars powered descent phase guidance design based on fixed-time stabilization technique," IEEE Transactions on Aerospace and Electronic Systems, pp. 1-10, 2018, DOI:10.1109/TAES.2018.2880051.

[6] M. Zapateiro, F. Pozo, H. R. Karimi, and N. Luo, "Semiactive control methodologies for suspension control with magnetorheological dampers," IEEE/ASME Transactions on mechatronics, vol. 17, no. 2, pp. 370380, 2012.

[7] Y. S. Wang and Y. Wang, "A gradient-based approach for optimal plant controller co-design," in American Control Conference, 2015, pp. 32493254.

[8] H. Zhu, C. K. Pang, and T. J. Teo, "Integrated servo-mechanical design of a fine stage for a coarse/fine dual-stage positioning system," IEEE/ASME Transactions on Mechatronics, vol. 21, no. 1, pp. 329-338, 2016.

[9] N. M. Wereley, "Integrated structure/control design of micropositioner for boring bar tool insert," Proceedings of SPIE - The International Society for Optical Engineering, vol. 12, no. 9, pp. 617-627, 2001.

[10] J. A. Perez, C. Pittet, D. Alazard, and T. Loquen, "Integrated control/structure design of a large space structure using structured $\mathrm{H}_{\infty}$ control," IFAC-PapersOnLine, vol. 49, no. 17, pp. 302-307, 2016.

[11] R. Chiang, J. Liu, and D. Bender, "Integrated robust control design methodology for an advanced s/c with large flexible structure," in AIAA Guidance, Navigation and Control Conference and Exhibit, 2013.

[12] Y. Zhang, D. Yang, and S. Li, "An integrated control and structural design approach for mesh reflector deployable space antennas," Mechatronics, vol. 35, pp. 71-81, 2016.

[13] J. Zhai, G. Zhao, and L. Shang, "Integrated design optimization of structural size and control system of piezoelectric curved shells with respect to sound radiation," Structural and Multidisciplinary Optimization, pp. $1-18,2017$.

[14] F. A. Shirazi, K. M. Grigoriadis, and D. Viassolo, "Wind turbine integrated structural and $\mathrm{lpv}$ control design for improved closed-loop performance," International Journal of Control, vol. 85, no. 8, pp. 11781196, 2012.

[15] S. F. Alyaqout, P. Y. Papalambros, and A. G. Ulsoy, "Combined robust design and robust control of an electric dc motor," IEEE/ASME Transactions on Mechatronics, vol. 16, no. 3, pp. 574-582, 2011.

[16] X. J. Lu and M. H. Huang, "Nonlinear-measurement-based integrated robust design and control for manufacturing system," IEEE Transactions on Industrial Electronics, vol. 60, no. 7, pp. 2711-2720, 2013.

[17] H. K. Fathy, J. A. Reyer, P. Y. Papalambros, and A. G. Ulsov, "On the coupling between the plant and controller optimization problems," in American Control Conference, 2001, pp. 1864-1869 vol.3.

[18] H. K. Fathy, P. Y. Papalambros, and A. G. Ulsoy, "Integrated plant, observer, and controller optimization with application to combined passive/active automotive suspensions," in Proceedings of the 2003 ASME, 2003, pp. 3375-3380 vol.4.

[19] H. K. Fathy, P. Y. Papalambros, A. G. Ulsoy, and D. Hrovat, "Nested plant/controller optimization with application to combined passive/active automotive suspensions," in American Control Conference, 2003, pp. 3375-3380 vol.4.

[20] B. Y. Kara and V. Verter, "Designing a road network for hazardous materials transportation," Transportation Science, vol. 38, no. 2, pp. 188-196, 2004. 
[21] P. Marcotte and G. Savard, "A bilevel modeling approach to pricing and fare optimization in the airline industry," Journal of Revenue and Pricing Management, vol. 2, no. 1, pp. 23-36(14), 2007.

[22] M. Kočvara and J. V. Outrata, "Effective reformulations of the truss topology design problem," Optimization and Engineering, vol. 7, no. 2, pp. 201-219, Jun 2006.

[23] X. Xu, W. Zhang, N. Li, and H. Xu, "A bi-level programming model of resource matching for collaborative logistics network in supply uncertainty environment," Journal of the Franklin Institute, vol. 352, no. 9, pp. 3873-3884, 2015.

[24] Q. Guo, J. Yin, T. Yu, and D. Jiang, "Saturated adaptive control of electrohydraulic actuator with parametric uncertainty and load disturbance," IEEE Transactions on Industrial Electronics, vol. 64, no. 10, pp. 7930$7941,2017$.

[25] S. Chang and T. Peng, "Adaptive guaranteed cost control of systems with uncertain parameters," IEEE Transactions on Automatic Control, vol. 17, no. 4, pp. 474-483, 1972.

[26] L. Yu, J. M. Xu, and Q. L. Han, "Optimal guaranteed cost control of singular systems with delayed state and parameter uncertainties," in American Control Conference, 2004, pp. 4811-4816 vol.5.

[27] D. Liu, D. Wang, F. Y. Wang, H. Li, and X. Yang, "Neural-networkbased online HJB solution for optimal robust guaranteed cost control of continuous-time uncertain nonlinear systems," IEEE Transactions on Cybernetics, vol. 44, no. 12, pp. 2834-2847, 2014.

[28] L. Yu and J. Chu, "An Imi approach to guaranteed cost control of linear uncertain time-delay systems," Automatica, vol. 35, no. 6, pp. 1155$1159,1999$.

[29] F. L. Lewis, D. Vrabie, and V. L. Syrmos, "Optimal control, 3rd edition," 2012.

[30] A. Kamadan, G. Kiziltas, and V. Patoglu, "Co-design strategies for optimal variable stiffness actuation," IEEE/ASME Transactions on Mechatronics, vol. 22, no. 6, pp. 2768-2779, 2017.

Tianyi Zeng Tianyi Zeng is with the School of Automation, Beijing Institute of Technology. He has received the M.S degree in Department of Control Science and Engineering from Harbin Institute of Technology, Heilongjiang, China, in 2015. His research interest covers motor driving system control and co-design of mechanism parameters and the controller.

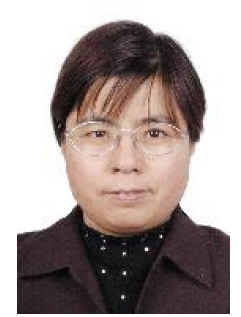

Xuemei Ren received the B.S. degree from Shandong University, Jinan, China, in 1989, and the M.S. and $\mathrm{Ph} . \mathrm{D}$. degrees in control engineering from the Beijing University of Aeronautics and Astronautics, Beijing, China, in 1992 and 1995, respectively. She has been a Professor at the School of Automation, Beijing Institute of Technology since 2002. From 2001 to 2002, and from April 2005 to October 2005, she visited the Department of Electrical Engineering, Hong Kong Polytechnic University, Hong Kong. From 2006 to 2007, she visited the Automation and Robotics Research Institute, University of Texas at Arlington, Arlington, TX, USA, as a Visiting Scholar. She has authored over 100 academic papers. Her current research interests include nonlinear systems, intelligent control, neural network control, adaptive control, multidrive servo systems, and time delay systems.

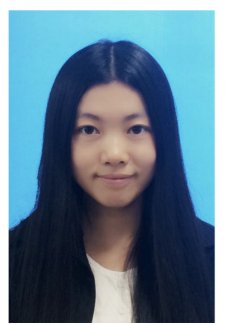

Yao Zhang received her Ph. D. in Department of Control Science and Engineering, Harbin Institute of Technology in 2018. She is currently a postdoctoral researcher in Queen Mary University of London. Her research interest covers sliding mode control, model predictive control and control applications.

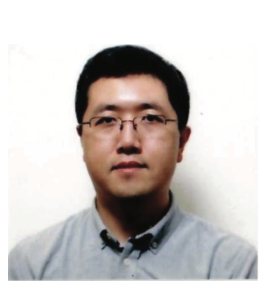

Guang Li received his Ph.D. degree in Electrical and Electronics Engineering, specialized in control systems, from the University of Manchester, in 2007. $\mathrm{He}$ is currently a senior lecturer in dynamics modelling and control in Queen Mary University of London, UK. His current research interests include constrained optimal control, model predictive control, adaptive robust control and control applications including renewable energies and energy storage, etc.

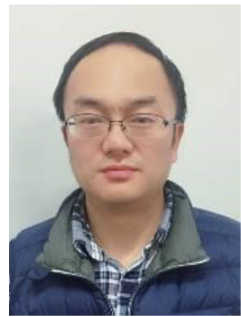

Jing Na received the B.Eng. and Ph.D. degrees in control engineering from the School of Automation, Beijing Institute of Technology, Beijing, China, in 2004 and 2010, respectively. From 2011 to 2013, he was a Monaco/ITER Postdoctoral Fellow with the ITER Organization, Saint-Paul-le's, Durance, France. From 2015 to 2017, he was a Marie Curie Intra-European Fellow with the Department of Mechanical Engineering, University of Bristol, U.K. Since 2010, he has been with the Faculty of Mechanical and Electrical Engineering, Kunming University of Science and Technology, Kunming, China, where he became a Full Professor in 2013. His current research interests include adaptive control, time-delay systems, neural networks, parameter estimation, repetitive control, and nonlinear control and applications. 